Association for Information Systems AIS Electronic Library (AISeL)

Wirtschaftsinformatik Proceedings 2005

Wirtschaftsinformatik

February 2005

\title{
Adaptive Design of Cross-Organizational Business Processes Using a Model-Driven Architecture
}

Bernhard Bauer

University of Augsburg

Stephan Roser

University of Augsburg

Jörg P. Müller

Siemens $A G$

Follow this and additional works at: http://aisel.aisnet.org/wi2005

\section{Recommended Citation}

Bauer, Bernhard; Roser, Stephan; and Müller, Jörg P., "Adaptive Design of Cross-Organizational Business Processes Using a ModelDriven Architecture" (2005). Wirtschaftsinformatik Proceedings 2005. 6.

http://aisel.aisnet.org/wi2005/6

This material is brought to you by the Wirtschaftsinformatik at AIS Electronic Library (AISeL). It has been accepted for inclusion in Wirtschaftsinformatik Proceedings 2005 by an authorized administrator of AIS Electronic Library (AISeL). For more information, please contact elibrary@aisnet.org. 
In: Ferstl, Otto K, u.a. (Hg) 2005. Wirtschaftsinformatik 2005: eEconomy, eGovernment, eSociety; 7. Internationale Tagung Wirtschaftsinformatik 2005. Heidelberg: Physica-Verlag

ISBN: 3-7908-1574-8

(C) Physica-Verlag Heidelberg 2005 


\title{
Adaptive Design of Cross-Organizational Business Processes Using a Model-Driven Architecture
}

\author{
Bernhard Bauer, Stephan Roser \\ University of Augsburg
}

Jörg P. Müller

Siemens AG

\begin{abstract}
To enable enterprises to keep up with the constant change in business relationships and cross-organizational value chains, it is crucial to develop adaptive business systems and value chains. In order to achieve this, methodologies, methods, and infrastructures to support end-to-end modeling of crossorganizational business processes are required, allowing changes to business processes being defined at the business level and providing well-defined (and possibly largely automated) model transformations and refinements down to the level of information and communication technology systems.

The contribution of this paper is threefold: First, we present a conceptual architecture for modeling collaborative business processes based on a model-driven architecture; second, we propose a design approach suitable to the model-driven architecture, and third, we provide two model transformations (mappings) to implement our design approach, thus enabling the smooth transition from an ARIS model via a computation-independent BPDM model to a platform-independent BPDM model.
\end{abstract}

Keywords: Business Process Modeling, Adaptive Value Networks, Model-Driven Architecture, Business Process Definition Metamodel, Cross-Organizational Business Processes, Collaborative Business Processes

\section{Introduction}

Over the past few years, enterprises have been undergoing a thorough transformation in reaction to challenges such as globalization, unstable demand, and mass customization. A key to maintain competitiveness is the ability of an enterprise to describe, standardize, and adapt the way it reacts to certain types of business events, and how it interacts with suppliers, partners, competitors, and customers. 
In the context of process orientation, enterprises today describe these procedures and interactions in terms of business processes, and invest huge efforts to describe and standardize these processes. The near future will bring an extension of these efforts towards cross-organizational business processes. Modelling and managing business processes that span multiple organizations involves new challenges, mainly regarding the ability to cope with change, decentralization, and the required support for interoperability. Parts of the work reported on in this paper are motivated from the European Integrated Project ATHENA [ATHENA]. ATHENA addresses the vision of seamless interoperation of distributed enterprises across and beyond Europe, focusing on the problem of interoperability ${ }^{1}$, but also covering aspects such as cross-organizational business process modeling and architectures and platforms for business process management and enactment. ATHENA addresses cross-organizational business processes at three related levels (business level, knowledge level and information and technology level).

The focus of this paper is on business process modeling, as opposed to run-time business process management. Speaking in terms of the ATHENA framework, its main contribution is at the business level. However, given the ultimate goal to support end-to-end business processes, by providing a process of gradual transition from abstract conceptual descriptions of business processes to concrete, executable business processes, requires us to consider the mapping to the information and communication technology level. Our approach towards this end is model-driven development and architecture (MDA) as promoted by the Object Management Group (OMG). Within MDA the software development process is driven by the activity of modeling the business software system. One of the major differences to traditional development processes lies in the nature of the artifacts that are created during the development process. These artifacts are formal models, i.e. models that can be understood by computers and finally be transformed into a representation that lends itself to execution which can effectively supported e.g. by a web services ICT infrastructure.

In this paper, starting from the ATHENA interoperability architecture, we propose a conceptual architecture for cross-enterprise business processes. Then, we present a methodical approach towards designing cross-enterprise business processes based on a model-driven architecture. The core contribution of the paper is a set of original mappings at and across different layers of the model-driven architecture. In particular, the model transformations we describe are: (1) Mapping from ARIS to an UML2 business process representation (adherent to the Business Process Definition Metamodel (BPDM) specification [Iyen04]) at the computationindependent model (CIM); (2) mapping from an UML2 representation at the CIM

1 In the context of ATHENA, interoperability is defined as "the ability of two or more systems or components to exchange information and to use the information that has been exchanged" [IDEAS]. 
level to an UML2 representation at the platform-independent model (PIM) (also adhering to BPDM).

\section{Background}

The next two sections outline the state-of-the-art in business-process related software architecture and IT infrastructure. It presents related work and standards in business process modeling, and additionally outlines the ATHENA interoperability definition and architecture underlying our work in section 0 .

\subsection{Model-Driven Architecture}

The Model Driven Architecture (MDA) (for details see [KlWB03]; this section is also based on this reference) is a framework for software development driven by the Object Management Group (OMG). The following three models are at the core of the MDA:

- Computation Independent Model (CIM): This is the most abstract model within the MDA. It describes business logic, business processes and workflows, and business requirements to a software system independent of computational technology.

- Platform Independent Model (PIM): This model is defined at a high level of abstraction; it is independent of any implementation technology. It describes a software system that supports some business. Within a PIM, the system is modeled from the viewpoint of how it best supports the business.

- Platform Specific Model (PSM): In the next step, the PIM is tailored to specify a system in terms of the implementation constructs available in one specific implementation technology. A PIM is transformed into one or more PSMs. For each specific technology platform a separate PSM is generated. Most systems today span several technologies; therefore it is common to have many PSMs with one PIM. The final step in the development is the transformation of each PSM to code. Because a PSM fits its technology rather closely, this transformation is relatively straightforward.

\subsection{Business Process Modeling}

The first generation of approaches to business process modeling [HuPo97] was using business charting tools such as PERT charts, flow charts, IDEF0, and data flow diagrams to graphically represent processes. With the exception of PERT charts, these diagrams were primarily used to represent the business processes that 
would be implemented by IT applications. However, once embedded in a computer program, the IT department would tend to own the process. For non-IT applications, most companies equated process specification with the writing of policy and procedure manuals. Such manuals were as hard to modify as the business logic encoded by application programs. This early form of separated IT and nonIT business process specification are often called the first wave of business process management.

In the early nineties, the second generation began with the likes of Hammer [Ham90] and Davenport [Dav93] advocating the notion of business process reengineering. One important development from this research were workflow management systems, i.e. the use of computers to automate the flow of documents among employees. During the same period, vendors of off-the-shelf software applications began to organize their application modules so that they could be represented as a business process. Here, one could diagram a business process by simply deciding how to link a number of application modules. Vendors such as SAP, PeopleSoft, and Oracle offered systems of this kind and were usually referred to as enterprise resource planning (ERP) systems.

Today, we are witness to the third generation of BPM. End-to-end business processes are the focus of internal and cross-company integration. Therefore business processes are freed from the limited perspectives of workflow management and ERP systems. Business processes are made the focus of all automation and business systems. Such a unification of IT and business becomes the new foundation upon which the enterprise is built - reducing the lag between management intent and execution.

To support such a vision, we need diagramming approaches that can adequately represent the process modelling requirements for third-generation business process management. The following main technologies address business process modelling:

- ARIS: The Architecture of integrated Information Systems (see [Sch98]) forms a framework for the development and optimization of integrated information systems. In this context the ARIS concept serves as model for creating, analyzing, and evaluating business management process chains. Thus ARIS allows the description of business processes and the complexity is reduced by decomposing them into different views. ARIS is commonly used by in specifying the business view of processes.

- EDOC: Enterprise Distributed Object Computing (EDOC) is an OMGsupported effort to simplify the development of component based systems by means of a modeling framework, based on UML 1.4 and conforming to the MDA. The business process modeling features of EDOC are now addressed by UML 2.0 diagrams, such as the Activity Diagram Component Diagram, and Composite Structure Diagram and therefore EDOC is not considered in this paper. 
- BPMN: The Business Process Modeling Notation (BPMN) specification, produced by BPMI (www.bpmi.org) provides a graphical notation for expressing business processes in a Business Process Diagram. The objective is to support process management by both technical users and business users by providing a notation that is intuitive to business users yet able to represent complex process semantics. UML 2.0 added many of the diagramming elements from BPMN to the UML 2.0 diagram family.

- UML2.0: The Unified Modeling Language is a language for visualizing, specifying, constructing and documenting software artifacts. It is a generalpurpose modeling language that can be used with all major object and component methods and applied to all application domains. Its extensive use has raised numerous application and implementation issues by modelers and vendors. UML 2.0 was produced to address many of these issues - including business process modeling [UML].

- BPDM: Resulting from an OMG Request for Submission, the primary objective of the Business Process Definition Metamodel (BPDM) initiative is to provide an abstract model for the definition of business processes (see [Iyen04]). BPDM is specified as a UML 2.0 profile enabling generic UML tools to both author or consume business models. As BPDM provides basic concepts from business process modelling, such as processes, tasks, rules, transactions, workers, and organizations, as first-class citizens, and additionally provides support for the modelling of collaboration, it appears a promising approach to combine the openness and genericity of UML with the expressiveness and vocabulary required for business process modelling. Mappings from a business-level model directly to runtime model like J2EE or BPEL4WS need to be defined and supported by tools. There are numerous activities towards this end, some of them carried through within the ATHENA project.

\section{The ATHENA Interoperability Architecture}

The ATHENA project [ATHENA] attempts to contribute towards the vision of seamless interoperation of distributed enterprises across and beyond Europe, focusing on the problem of interoperability, but also addressing aspects such as cross-enterprise business process modeling as well as architectures and platforms for business process management. Figure 1 illustrates the conceptual architecture of ATHENA. The architecture addresses cross-organizational interoperability at three related levels: The business level, the knowledge level, and the information and communication technology (ICT) level. At the business level, all issues related to the organization and the operations of an enterprise are addressed, i.e. the way an enterprise is organized, how it operates to produce value, how it manages its relationships, etc.. Interoperability at business level is the organizational and op- 
erational ability of an enterprise to cooperate with other organizations. The knowledge level deals with acquiring a deep and wide knowledge of the enterprise. This includes knowledge of internal aspects such as products or the way the administration operates and controls as well as knowledge of external aspects such as partners and suppliers or laws and regulations. Furthermore, speed of changes tends to increase and the knowledge of the environment, in its widest accepted meaning, becomes more important, and sometimes even vital for the success of the business. Finally, the ICT Systems level focuses on the ICT solutions that allow an enterprise to operate, make decisions, and exchange information within and outside its boundaries. Interoperability at ICT Systems level should be seen as the ability of an enterprise's ICT systems to cooperate with those of other, external organisations.

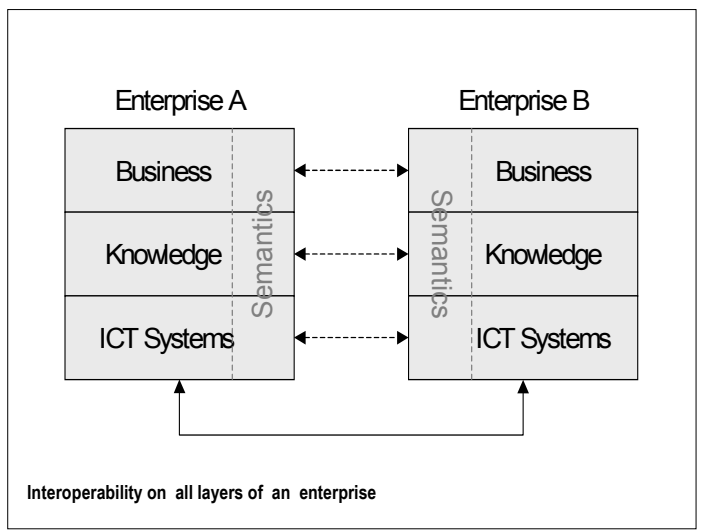

Figure 1: The ATHENA business interoperability architecture (cp. IDEAS road map [IDEAS])

In this paper, we focus on the business and ICT systems level. We regard the knowledge level as somewhat orthogonal to business and ICT systems level, in that different abstractions and representations of knowledge will be required at business and ICT level respectively.

In the following section, we discuss options for a conceptual modeling architecture before we describe our methodical approach and the MDA mappings in Section 5 . 


\section{Modeling Architecture for Cross-Organizational Business Processes}

In order to enable business processes to collaborate with partners and to facilitate the composition of business processes, the paradigm of service-oriented architecture is applied to business process modeling [FrGJ04]. Business processes and activities are treated as components which provide services to and consume services from other business process components. Interacting business processes form a network of interconnected processes where conversations are conducted. The same process can appear in multiple solutions and can be connected to different partners in each case. The service-oriented approach to business process modeling is appealing mainly because it provides a natural logical view to distributed business systems and at the same time naturally lends itself to a mapping into an ICT perspective.

[Pel03] suggests employing the terms orchestration and choreography to describe the collaboration between service components.

- Orchestration: Orchestration refers to an executable process that may interact both with internal and external services. Orchestration describes the interactions between services, including the business logic and the execution order of the interactions. With orchestration, the process is always controlled from the perspective of one of the business parties.

- Choreography: Choreography describes processes in a more collaborative way, where each party involved in the process describes the part it plays in the interaction. Choreography tracks the sequence of messages exchanged between multiple business parties. Often choreography is associated with the public message exchange that occurs between multiple services.

Orchestration differs from choreography in describing process flow between service components, controlled by a single party (as we can see in figure 2 the global process is often the controlling party). More collaborative in nature, choreography describes the sequence of public messages, where no party owns the conversation by contolling the process flow (see figure 2 ). 


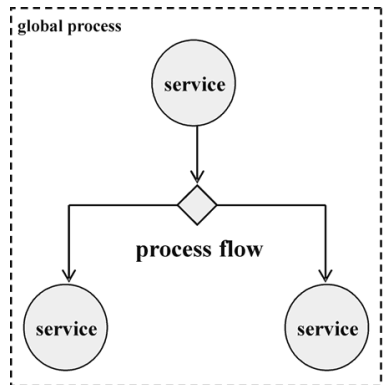

Service Orchestration

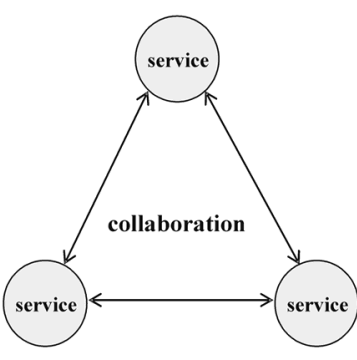

Service Choreography

Figure 2: Service orchestration and service choreography [Pel03]

[FrGJ04] distinguishes between an internal and an external view of business processes. Depending on the viewpoint, a process is described either as an executable, abstract, or collaborative process.

- Executable Process: The internal view models the 'how' of a business process to a level of detail the modeler knows. In [IBM] processes, which model process flows as a set of partially ordered tasks, are called executable processes. As the flow of the processes' interactions is described from the point of view of a single process, which coordinates participating sub-process, this kind of process composition is referred to as process orchestration.

- Abstract process: The external view models the 'what' of a business process. Each process specifies its roles, which it takes up in the collaboration with other processes, but doesn't give any indication about its own realization. The interfaces of such business processes components are called abstract processes. Abstract processes additionally describe their public interactions they perform in relation to their roles in collaborations. They give no indication about whole collaborations or their own realization.

- Collaborative process: In the case of process choreography the collaboration between abstract processes is described in collaborative processes. Collaborative processes use abstract processes to model the message exchange between processes and the sequence of the message exchange from the viewpoint of an external observer. The collaborations between the involved parties are modeled as interaction patterns between their roles they take in.

Business protocols can be realized in multiple ways, which differ in how the business protocols' conversation flow is coordinated. Our architecture for modeling cross-enterprise business processes relies on the broker approach. An intermediary acts as a global observer process coordinating the partners, which take part in the cross-enterprise business process. There are several reasons for applying a broker pattern: 
- Without a broker one executable process per partner has to be implemented, which coordinates the partners' activity in the business protocol. This is problematic since control flow logic of one cross-enterprise business process is divided into different executable process. Due to the mutual exchange of messages these processes depend on one another. Changing the business protocol would result in changing multiple executable processes.

- This disadvantage doesn't appear by implementing a broker coordinating the conversation between the communication partners. Each business protocol is realized through one of the broker's executable processes. Moreover this approach is more convincing, since cross-enterprise business process, i.e. collaborative processes, are modeled as separate processes from the viewpoint of an external observer.

\section{Model-Driven Business Process Design}

In this section, we describe the core contributions of this paper: A methodical approach to designing business processes via model-driven architecture and two mappings that implement essential parts of the model-driven approach.

\subsection{Methodical Approach}

Due to the fact that different kinds of experts, like economists and computer scientists, did business process modeling from different angles, the existence of different approaches is not surprising. In addition to the differences in the models used, we can distinguish between a top-down and a bottom-up approach in the context of $\mathrm{MDA}^{2}$ :

- In the top-down approach business processes are first modeled in a computational independent fashion. Corporate as well as cross-enterprise business processes are modeled by process consultants with a business background. A very common modeling methodology for this kind of business process modeling is ARIS.

- Computer scientists tend to choose a more bottom-up approach to business process modeling, starting from platform specific models, which allow automated process execution (e.g. BPEL4WS, BPML, etc.). Hence models for higher level descriptions of business processes, which can be mapped to those

2 In this context top-down means to start modeling with less formal models, which represent the real business processes very well. A bottom-up approach would use formal models, which e.g. can be executed by computers, but sometimes lack of relevant concepts for modeling more advanced business processes. 
process execution languages, like the Business Process Definition Metamodel (BPDM) are emerging. Since for most IT systems object-oriented methods have become accepted as a standard, it is obvious that these systems are modeled in UML. In addition, UML offers the advantage of openness and (to a large extent) vendor independence.

In such an environment it is important for developers of IT systems to ensure that the ARIS models of economists are consistent with the BDPM models of computer scientists. The change of modeling methods is a very crucial point in the development of an IT system, since mistakes in process modeling made here are rarely found before the system is deployed.

In this paper we introduce a mapping between ARIS and BPDM. This mapping makes it possible to deduce BPDM-Models at PIM level from ARIS-Models at CIM-level. Processes at PIM level shall be described in such a way, that they can be transformed to process execution languages on PSM level. The choice of an UML-based business process representation at the CIM and PIM levels addresses the tendency towards improving interoperability through choosing open standards for business process representation. At the same time, supporting a mapping from ARIS into BPDM takes the fact into account that ARIS is the leading modelling business process methodology in industry and tries to improve the impact of the work done in ATHENA by widening its scope, hence making it easier for enterprises to migrate their business process models into a model-driven framework. Finally, choosing BPDM as a vendor-independent representation will allow to leverage the support for modeling collaboration which will be provided in BPDM and makes our approach particularly suitable for the modeling of decentralized, cross-enterprise processes.

The mapping is performed in two stages:

- Transformation of ARIS models to object-oriented BPDM models, which are as far as possible equivalent, whereas static and dynamic structures have to be considered. The focus is on the transformation of the process-oriented eventdriven process chains, which are used for the representation of dynamic aspects in ARIS.

- Generation of BPDM models at PIM level out of BPDM models at CIM level. Tools which implement the MDA approach will provide implementations to perform this task automatically. 


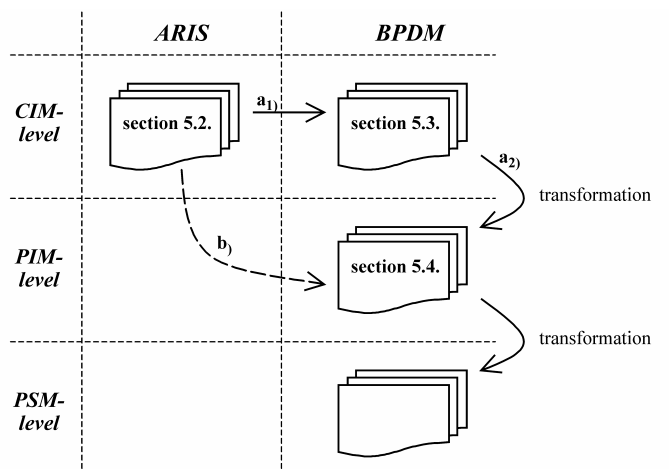

Figure 3: Model-driven business process design approach

As illustrated in Figure 3, there are two alternatives in developing the ARISBPDM mapping. In alternative a) each of the two identified tasks are carried out in a separate step. Alternative b) suggests performing the mapping, i.e. both the transformation from ARIS to BPDM and generation of PIM models from CIM models, in a single step. In the following, we shall briefly discuss advantages and disadvantages of these alternatives.

An advantage of alternative b) is that the mapping is conducted in one single step. It avoids the development and therefore the existence of a (redundant) second model at CIM level. Though it would be possible to realize the mapping in one single step, there are a number of aspects we consider problematic. A parallel and nested execution of the mapping, in reference to the mapping of ARIS to BPDM and the transformation of CIM level to PIM level, leads to a large, monolithic and poorly comprehensible mapping. Moreover, two different and insufficiently integrated modeling languages are used for describing the development of one ICT system, though integrating different models is one of the main points required by the MDA.

These disadvantages do not occur in alternative a), where the mapping is separated into two tasks as described above. Hence, the mapping becomes more transparent and easier to understand for the user. It is easier to track which model elements have their origin in ARIS diagrams and which model elements were generated through the transformation to PIM level. Furthermore, it is possible to use completely integrated models for system development. Even the existence of two models for one system at CIM level need not be a disadvantage since different models may appeal to different types of users (i.e. ARIS to business users and BPDM to users with an ICT perspective).

For the development of an ARIS-BPDM mapping we choose alternative a) and sum up the correlations and differences between various model types. ARIS models are common for describing corporations and their business processes from an economical point of view at CIM level. With BPDM models system developers 
describe the business processes and corporation's aspects from an object-oriented point of view at CIM level. At PIM level BPDM models describe business processes and aspects of the corporation, which are relevant for the ICT system in development, in a more detailed way. Finally on PSM level runtime models, like for example automatable business processes models, are described.

In the remainder of this section we present a mapping from ARIS models at CIM level to BPDM models at PIM level ${ }^{3}$. The mapping is showed by an example. The example follows the architecture for modeling cross-enterprise business processes in Section 0. Since the focus of the mapping is the transformation from processoriented to object-oriented descriptions of the control flow, the examples cover only models which are relevant for the description of control flows.

\subsection{ARIS Model at CIM Level}

Process structures can be modeled as value-added chain diagrams (VACDs) in ARIS. The control flow is modeled by event-driven process chains (EPCs) attached to the respective processes. EPCs consist of functions representing tasks which contribute to the corporation's objectives; events representing states, which are conditions under which a function can be executed or hold when a function was terminated; process interfaces indicating the passing of the control flow from one process to another one. Therefore a process interface at the beginning of an EPC specifies the process types by which the process can be invoked. Process interfaces at the end of an EPC specify which process types the process intends to invoke.

In the example (see Figure 4) the processes of a buyer and a seller conducting cross-enterprise business processes are modeled. The buy process is partitioned into an identify demand and a verify offer process. The sell process consists of a calculate offer and a coordinate offerings process.

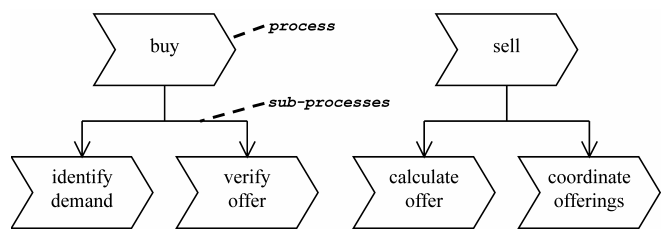

Figure 4: Example of an ARIS value-added chain diagram

Figure 5 shows the refined EPCs for the example processes. When demand is identified by the buyer, the identify demand process finally invokes the seller's calculate offer process. This process calculates an offer and returns the result to

3 The mapping of executable and abstract processes as well as static process structure has been implemented in a protoype. 
the buyer. There the verify offer process decides whether the offer can be accepted or not. When the offer is accepted the process informs the seller's coordinate offerings process about this. In the case the offer cannot be accepted, the verify offer process can invoke the calculate offer process for a new offer or inform the coordinate offerings process that the offer is refused.

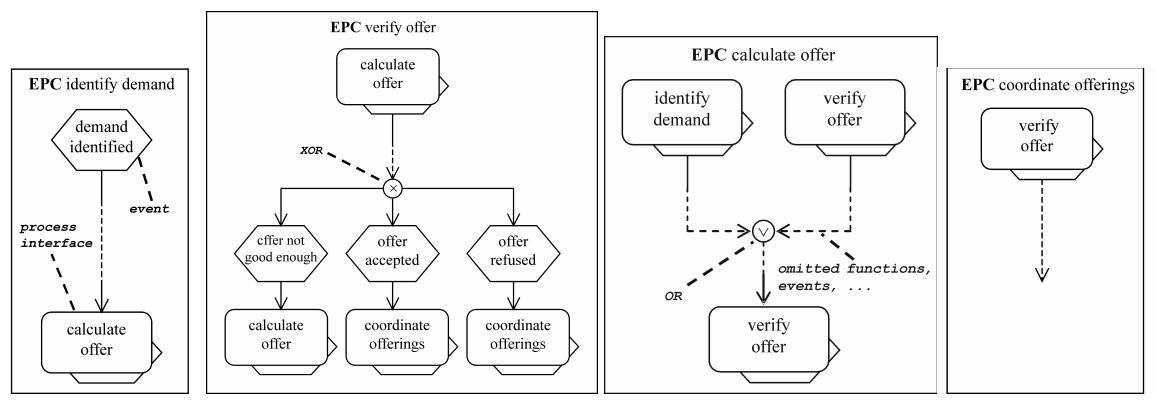

Figure 5: Example of ARIS event-driven process chains

\subsection{Mapping to BPDM Model at CIM Level}

Since BPDM is an extension of UML2 it is allowed to use UML2 concepts in BPDM models. In BPDM we model static aspects in class diagrams. Dynamic aspects of the collaboration between the processes of the buyer and the seller are modeled as collaborative processes and business protocols respectively. Therefore we use sequence diagrams. Additonally we apply interaction overview diagrams to model the external view on the enterprises' processes as abstract processes.

Mapping rules for modeling static aspects are as follows:

\begin{tabular}{|l|l|}
\hline name of rule & Rule CIM CD Process \\
\hline ARIS-concept & BPDM-concept \\
\hline function & class with stereotype «process» \\
\hline
\end{tabular}

Table 1. Rule for mapping processes

\begin{tabular}{|l|l|l|}
\hline name of rule & Rul_CIM_CD processhierarchy \\
\hline & ARIS & BPDM \\
\hline $\begin{array}{l}\text { assoziation- } \\
\text { type }\end{array}$ & , is process-oriented superior & $\begin{array}{l}\text { assoziation (directed from } \\
\text { source to destination) }\end{array}$ \\
\hline source-object & function (VACD) & class with stereotype «process» \\
\hline $\begin{array}{l}\text { destination- } \\
\text { object }\end{array}$ & function (VACD) & $\begin{array}{l}\text { class with stereotype «process» } \\
\text { (role , subProcess ') }\end{array}$ \\
\hline
\end{tabular}

Table 2. Rule for mapping process hierachy 


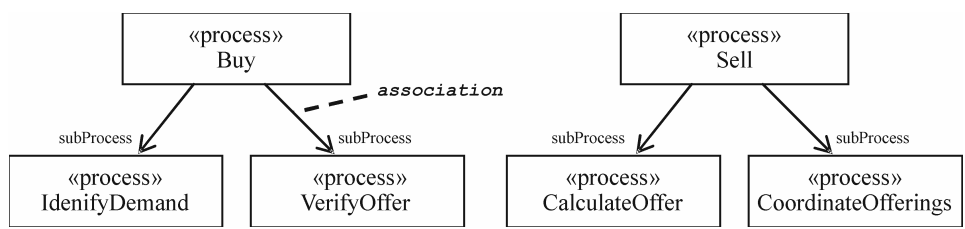

Figure 6: BPDM - class diagram at CIM level

For the dynamic aspects described in the ARIS-model we have to generate diagrams for executable, abstract and collaborative processes in BPDM. Since the focus is on collaboration between processes we omit the mapping of executable processes in this paper ${ }^{4}$. We concentrate on the mapping of abstract processes and on the description of the collaborative processes.

First, we present an excerpt of the main mapping rules for abstract processes.

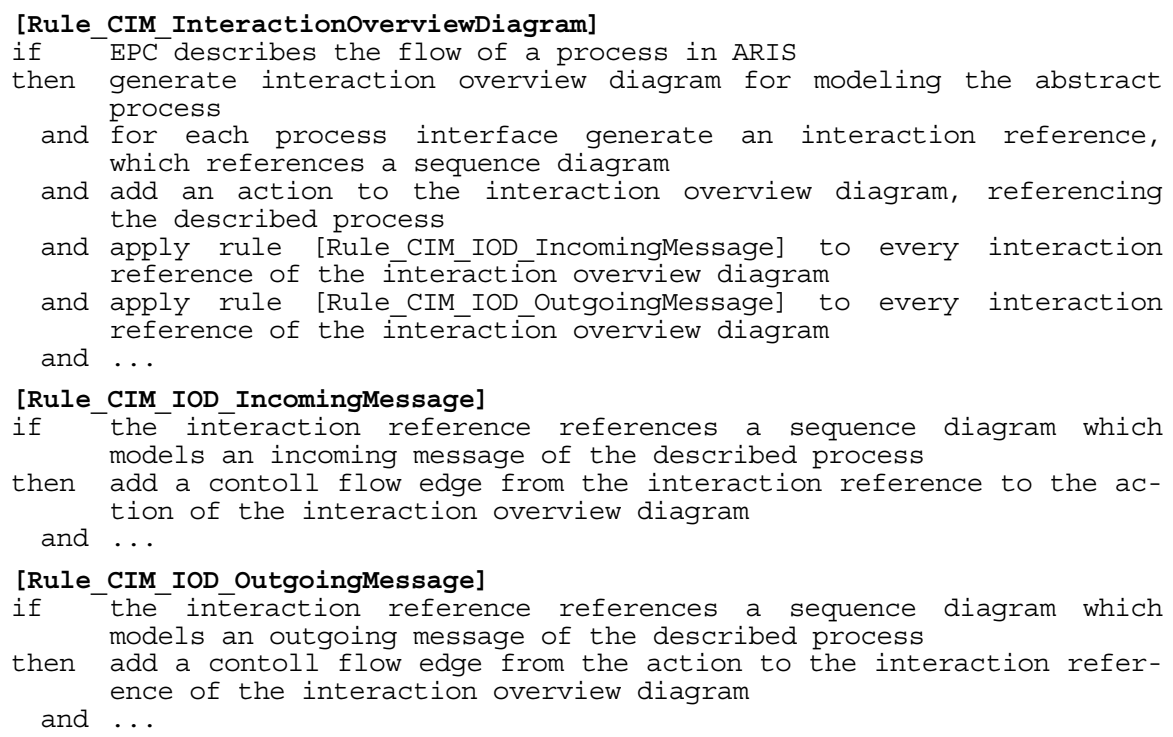

$4 \quad$ A mapping for executable processes from ARIS to BPDM has already been specified and implemented in a prototype. 

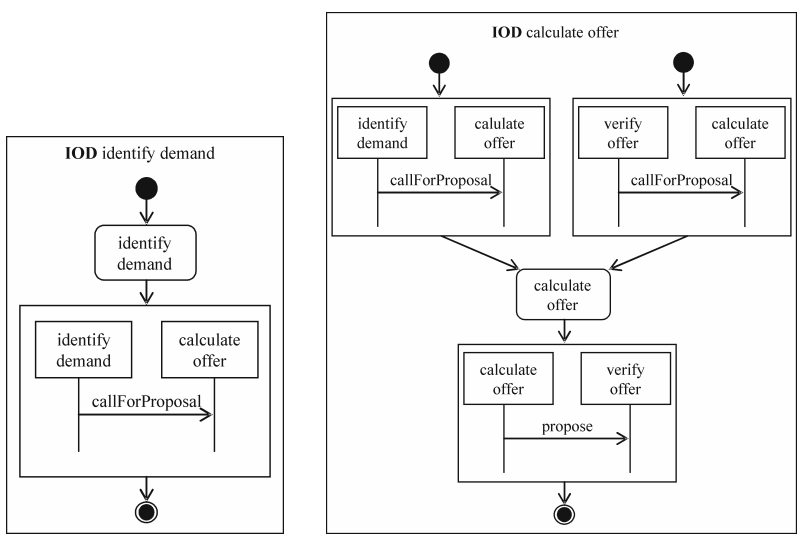

Figure 7: BPDM - Abstract process at CIM level

In Figure 7 the abstract processes verify offer and calculate offer can be seen. There the exchanged messages' order of one process is model as an activity diagram. The calculate offer process for example receives a callForProposalmessage from the identify demand or verify offer process. After the internal process flow (described in the executable process) has been executed, a proposemessage is sent to the verify offer process.

While information about the cross-enterprise processes in the ARIS model is contained in the EPC, i.e. through process interfaces, we use sequence diagrams to represent the business protocol interactions in UML. Unlike in the previous parts this part of the mapping has to be conducted manually, since it is not formalized yet. In Figure 8 the corresponding business protocol is modeled as the MyContractNet Protocol.

- The processes (agents) representing buyer and seller respectively conduct a negotiation. They are modeled as lifelines.

- In the sequence diagram internal behavior of e.g. the seller process is not visible.

- Messages are derived from the process interfaces of the EPCs in ARIS. Parameters of the messages, which are not visible in the discussed ARIS diagrams, are added to obtain a more complete model.

- Decisions in the control flow are mapped to combined fragments, e.g. the alternative fragment which models whether an offer is accepted or not.

- Guards are derived from the events in the EPCs and specify conditions for the alternatives to be taken. 


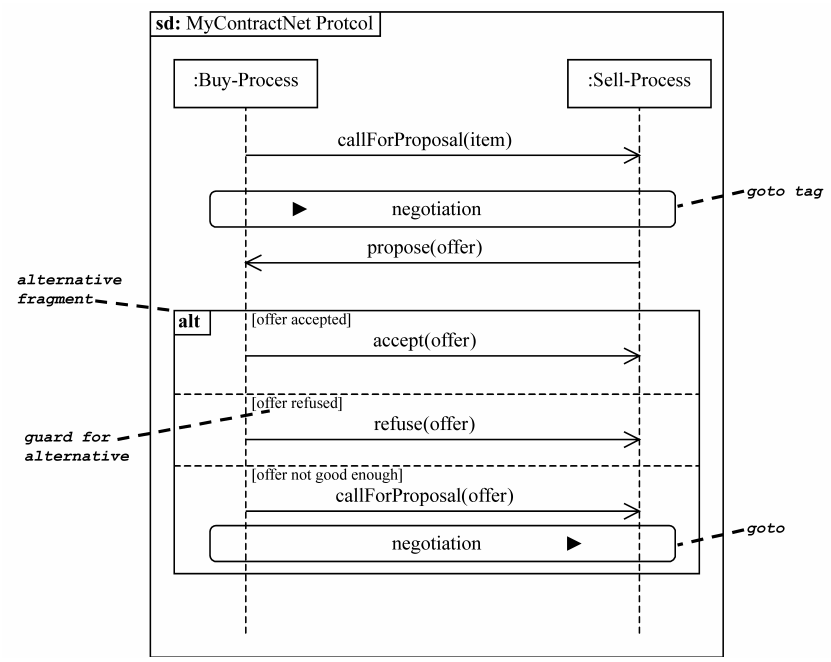

Figure 8: BPDM - business protocol modeled in sequence diagram

\subsection{BPDM Model at PIM Level}

At PIM level business processes und business protocols are modeled from the IT implementation point of view. According to our modeling architecture of crossenterprise business processes above, the collaborative process is realized through a broker process (see Figure 9) coordinating the control flow of the business protocols.

Figure 9: BPDM - process class of the broker

For modeling the broker process's internal behavior as an executable process we use an activity diagram. Following constructs can be found in the activity diagram:

- The control flow identifies sequencing of activities. Data flow identifies the flow of data objects between activities. Data objects being in- and outputs for actions are modeled as PINs. The names of the PINs specify the type of the data objects exchanged.

- BPDM [FrGJ04] describes actions stereotyped «send» or «receive» to model inter-process communication. The corresponding partner processes are identified by activity partitions in which these actions are modeled. 
- Decision nodes and merge nodes are used to model alternatives of the control flow. Which alternatives have to be taken is specified with guards.

Although the activity diagram models the internal view of the broker process, i.e. the executable process, elements used to model the external view of a process are also present, e.g. the stereotyped actions are also used to describe abstract processes.

In Figure 10, the MyContractNet protocol is depicted in an activity diagram as an executable process. We give a brief description of main tasks performed by transforming the sequence to an activity diagram:

- Lifelines of a sequence diagram become activity partitions in an activity diagram.

- Sequence diagram messages lead to stereotyped actions in an activity diagram. In the example the initial receive action initMyContractNet starts the broker's process. Due to the callForProposal-message of the sequence diagram the broker process sends a callForProposal to the sell agent's process. Since the callForProposal-message contains the parameter item, a data flow between the two actions is modeled. As we use asynchronous messaging, the callForProposal-message's results, i.e. an order, are passed to the broker's process in the next receive action callForProposal_CB. The broker's process sends the propose to the sell agent's process.

- The alternative fragment of a sequence diagram, which models whether an order is accepted or not, is realized as a decision node in the activity diagram.

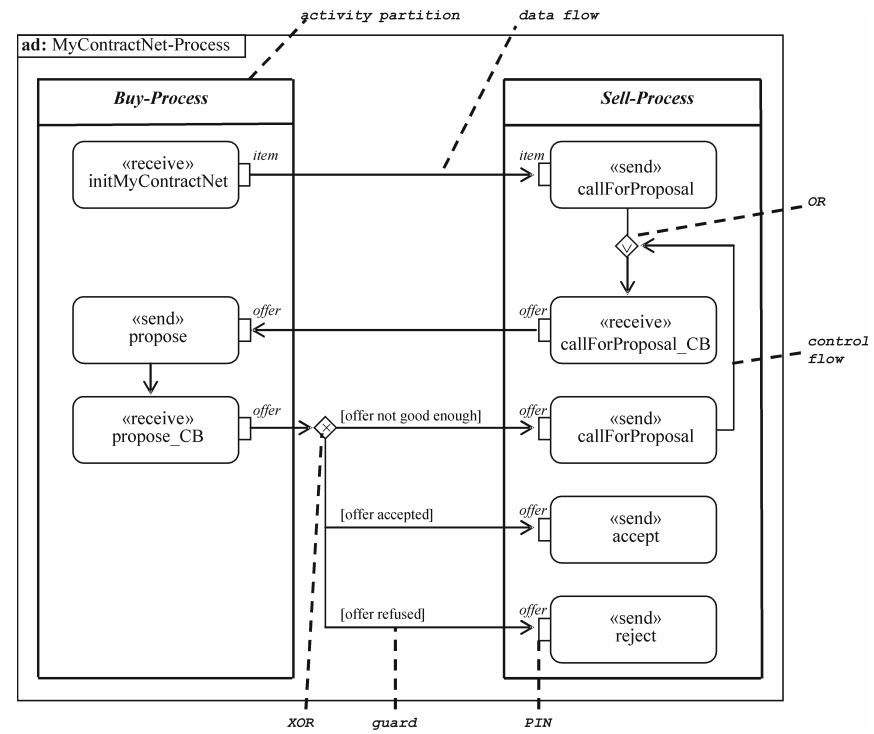

Figure 10: BPDM - modeling cross-enterprise business process control flow in an activity diagram 


\section{Conclusions and Outlook}

To enable enterprises to keep up with the constant change in business relationships and cross-organizational value chains, it is crucial to develop adaptive business systems and value chains. In order to achieve this, methodologies, methods, and infrastructures to support end-to-end modeling of cross-organizational business processes are required, allowing changes to business processes being defined at the business level and providing well-defined (and possibly largely automated) mappings and refinements down to the level of ICT systems. The contribution of this paper is threefold: First, we present a conceptual architecture for modeling cross-organizational business processes based on a model-driven architecture; second, we propose a design approach suitable to MDA, and third, we provide specifications of two model transformations (mappings) to implement the design approach, thus enabling the smooth transition from an ARIS model via a computation-independent BPDM model to a platform-independent BPDM model.

Future work will complete the approach by adding mappings to selected platformspecific models including the BPEL4WS language [IBM]. In addition, we will explore richer models of cross-organizational business processes, such as that proposed by [SchOr01], which will allow us to address a wider range of central and decentral collaboration architectures. A third strand of future work will be to enhance adaptability of runtime process infrastructures by a Robust Planning and Execution Layer that will combine abilities for dynamic service discovery, support for richer transaction and compensation in business process execution, and dynamic service composition. Preliminary work in this direction has been published in [MuBF04] and [BaMR04].

\section{Acknowledgements}

One author contributing to the work reported in this paper has been funded by the ATHENA IP under the European grant FP6-IST-507849. It does not represent the view of E.C. nor that of other consortium members, and authors are responsible for the paper's content.

\section{References}

[ATHENA] ATHENA. Advanced Technologies for Interoperability of Heterogeneous Enterprise Networks and their Applications. FP6-IST-507849. http://www.athena-ip.org 
[BaMR04] Bauer, Müller, Roser. A Model-driven Approach to Designing Cross-Enterprise Business Processes, MIOS, 2004

[Dav93] Davenport. Process Innovation. Reengineering Work through Information technology. Boston, 1993

[FrGJ04] Frank, Gardner, Johnston. Business Process Definition Metamodel - Concepts and Overview, IBM, 2004

[Ham90] Hammer. Reengineering Work: Don't automate, Obliberate. Harvard Business Review, 68, 1990, pp. 104-112

[HuPo97] Huber, Poestges. Geschäftsprozeßmanagement. Prinzipien und Werkzeuge für ein erfolgreiches Gestalten von Geschäftsprozesssen. In: Corsten, H.: Management v. Geschäftsprozessen. Theoretische Ansätze - Praktische Beispiele. Stuttgart, 1997

[IBM] IBM. Business Process Execution Language for Web Services Version 1.1, http://www.ibm.com/developerworks/library/ws-bpel/

[IDEAS] IDEAS. Interoperability Developments for Enterprise Application and SoftwareRoadmaps. European Project IST-2001-37368. http://www.ideas-roadmaps.net

[Iyen04] Iyengar et al. Business Process Definition Metamodel. Revised Submission to BEI RFP bei/2003-01-06. 2004

[KlWB03] Kleppe M., Warmer J., Bast W. MDA Explained - The Model Driven Architecture: Practice and Promise, Addison Wesley, 2003

[MuBF04] Müller, Bauer and Friese. Programming software agents as designing executable business processes: a model-driven perspective. Vol. 3067 of Lecture Notes in AI, Springer, 2004

[Pe103] Peltz. Web Service Orchestration and Choreography, Web Service Journal Volume3 Issue 7, 2003

[Sch98] Scheer. ARIS - Modellierungsmethoden, Metamodelle, Anwendungen, Springer, 1998

[SchOr01] Schulz, Orlowska. Architectural Issues for Cross-Organisational B2B Interactions, International Workshop on Distributed Dynamic Multiservice Architectures at ICDCS-21, IEEE Computer Society Press, 2001

[UML] UML Homepage. The Object Management Group. http://www.omg.org/uml/

[W3C] W3C Web Service glossary. http://www.w3.org/TR/ws-gloss/ 
